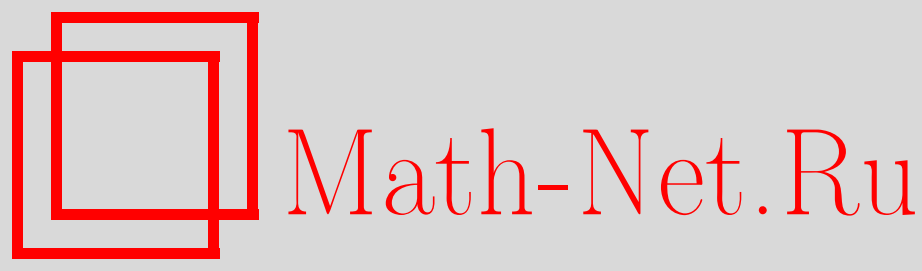

Л. В. Богданов, Интерполирующие дифференциальные редукции многомерных интегрируемых иерархий, $Т М \Phi$, 2011, том 167, номер 3, 354-363

DOI: https://doi.org/10.4213/tmf6646

Использование Общероссийского математического портала Math-Net.Ru подразумевает, что вы прочитали и согласны с пользовательским соглашением http://www . mathnet.ru/rus/agreement

Параметры загрузки:

IP : 54.166 .219 .16

26 апреля 2023 г., $12: 44: 33$

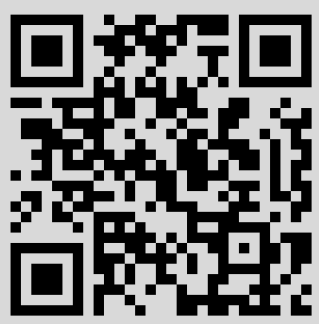




\title{
ИНТЕРПОЛИРУЮЩИЕ ДИФФЕРЕНЦИАЛЬНЫЕ РЕДУКЦИИ МНОГОМЕРНЫХ ИНТЕГРИРУЕМЫХ ИЕРАРХИЙ
}

\begin{abstract}
Схема конструирования дифференциальных редукций, предложенная недавно для иерархии Манакова-Сантини, применена к общему многомерному случаю. Более подробно рассмотрен четырехмерный случай, связанный со вторым "небесным" уравнением и его обобщением, предложенным Дунайским. Приведена характеризация дифференциальных редукций в терминах уравнений Лакса-Сато, а также в рамках метода одевания, основанного на нелинейной задаче Римана-Гильберта.
\end{abstract}

Ключевые слова: многомерные интегрируемые иерархии, дифференциальные редукции, уравнения Плебаньского.

\section{1. ВВЕДЕНИЕ}

В работе [1] мы построили класс редукций иерархии, ассоциированной с системой

$$
\begin{aligned}
& u_{x t}=u_{y y}+\left(u u_{x}\right)_{x}+v_{x} u_{x y}-u_{x x} v_{y} \\
& v_{x t}=v_{y y}+u v_{x x}+v_{x} v_{x y}-v_{x x} v_{y}
\end{aligned}
$$

введенной в статье [2] (см. также работы [3], [4]). В работе [5] было показано, что простая дифференциальная редукция $\alpha u=v_{x}$, где $\alpha=$ const, системы Манакова-Сантини (1) соответствует интерполирующей системе, которая была введена в работе [5] как "наиболее общая симметрийная редукция второго уравнения heavenly по конформному вектору Киллинга с нулевой автодуальной производной". Термин "интерполирующая" связан с тем фактом, что система (1) с указанной редукцией осуществляет "интерполяцию" между бездисперсионным уравнением КП, возникающим при $\alpha \rightarrow 0$, и системой, рассмотренной в работе [6] (см. также [7]-[9]), которая возникает при $\alpha \rightarrow \infty$. Используя формулировку иерархии Манакова-Сантини в терминах уравнений Лакса-Сато [10], [11], в работе [1] мы ввели класс редукций, в котором редукция нулевого порядка соответствует бездисперсионной иерархии КП, а редукция первого порядка дает иерархию, связанную с интерполирующей системой. Мы

${ }^{*}$ Институт теоретической физики им. Л.Д. Ландау РАН, Черноголовка, Московская обл., Россия. E-mail: leonid@landau.ac.ru 
привели производящее уравнение и форму Лакса-Сато редуцированной иерархии для интерполирующей системы, а также для редукции произвольного порядка. Редукция любого порядка $k$ содержит параметр $\alpha$ и является в каком-то смысле интерполирующей, давая бездисперсионную иерархию КП при $\alpha \rightarrow 0$ и редукцию Гельфанда-Дикого порядка $k$ для иерархии Манакова-Сантини при $\alpha \rightarrow \infty$. Важно заметить, что редукция Гельфанда-Дикого для иерархии Манакова-Сантини не влечет стационарность по высшему времени. Была также дана характеризация редукций в терминах данных одевания.

В настоящей статье мы переносим конструкцию, развитую в работе [1], на случай общей $(N+2)$-мерной иерархии [10], [11]. Иерархия Манакова-Сантини соответствует $N=1$, это случай не общего положения, а в общем случае присутствуют некоторые новые черты. Мы уделяем особое внимание случаю $N=2$, который содержит второе "небесное" уравнение Плебаньского [12] и его обобщение, предложенное Дунайским [13].

Интересно отметить глубокое структурное сходство нашей конструкции с картиной дифференциальных редукций в стандартном (дисперсионном) интегрируемом случае [14]-[17], несмотря на то что технические средства существенно отличаются.

\section{2. ИЕРАРХИЯ}

Иерархия Манакова-Сантини представляет собой частный случай, соответствующий $N=1$, общей $(N+2)$-мерной иерархии, определяемой производящим соотношением

$$
\left(J_{0}^{-1} d \Psi^{0} \wedge d \Psi^{1} \wedge \cdots \wedge d \Psi^{N}\right)_{-}=0,
$$

где $\Psi^{0}, \ldots, \Psi^{N}-$ ряды вида

$$
\begin{aligned}
\Psi^{0} & =\lambda+\sum_{n=1}^{\infty} \Psi_{n}^{0}\left(\mathbf{t}^{1}, \ldots, \mathbf{t}^{N}\right) \lambda^{-n}, \\
\Psi^{k} & =\sum_{n=0}^{\infty} t_{n}^{k}\left(\Psi^{0}\right)^{n}+\sum_{n=1}^{\infty} \Psi_{n}^{k}\left(\mathbf{t}^{1}, \ldots, \mathbf{t}^{N}\right)\left(\Psi^{0}\right)^{-n},
\end{aligned}
$$

$1 \leqslant k \leqslant N, \mathbf{t}^{k}=\left(t_{0}^{k}, \ldots, t_{n}^{k}, \ldots\right)$ и $(\cdot)_{-}$- проекция на отрицательные степени $\lambda$. В соотношении (2) $J_{0}$ - детерминант матрицы Якоби $J$,

$$
J_{0}=\operatorname{det} J, \quad J_{i j}=\partial_{i} \Psi^{j}, \quad 0 \leqslant i, j \leqslant N,
$$

где $\partial_{0}=\partial / \partial \lambda, \partial_{k}=\partial / \partial x^{k}, x^{k}=t_{0}^{k}, 1 \leqslant k \leqslant N$.

Производящее соотношение (2) эквивалентно набору уравнений Лакса-Сато

$$
\partial_{n}^{k} \boldsymbol{\Psi}=\sum_{i=0}^{N}\left(\left(J^{-1}\right)_{k i}\left(\Psi^{0}\right)^{n}\right)_{+} \partial_{i} \boldsymbol{\Psi}, \quad 0 \leqslant n \leqslant \infty, \quad 1 \leqslant k \leqslant N,
$$

где $\boldsymbol{\Psi}=\left(\Psi^{0}, \ldots, \Psi^{N}\right),(\cdot)_{+}-$проекция на неотрицательные степени $\lambda$. Первые потоки иерархии имеют вид

$$
\partial_{1}^{k} \boldsymbol{\Psi}=\left(\lambda \partial_{k}-\sum_{p=1}^{N}\left(\partial_{k} u_{p}\right) \partial_{p}-\left(\partial_{k} u_{0}\right) \partial_{\lambda}\right) \boldsymbol{\Psi}, \quad 0<k \leqslant N,
$$


где $u_{0}=\Psi_{1}^{0}, u_{k}=\Psi_{1}^{k}, 1 \leqslant k \leqslant N$. Условие совместности любой пары линейных уравнений (например, с $\partial_{1}^{k}$ и $\partial_{1}^{q}, k \neq q$ ) приводит к замкнутой нелинейной $(N+2)$-мерной системе уравнений в частных производных для функций $u_{k}, u_{0}$, которая может быть записана в виде

$$
\begin{gathered}
\partial_{1}^{k} \partial_{q} \hat{u}-\partial_{1}^{q} \partial_{k} \hat{u}+\left[\partial_{k} \hat{u}, \partial_{q} \hat{u}\right]=\left(\partial_{k} u_{0}\right) \partial_{q}-\left(\partial_{q} u_{0}\right) \partial_{k}, \\
\partial_{1}^{k} \partial_{q} u_{0}-\partial_{1}^{q} \partial_{k} u_{0}+\left(\partial_{k} \hat{u}\right) \partial_{q} u_{0}-\left(\partial_{q} \hat{u}\right) \partial_{k} u_{0}=0
\end{gathered}
$$

где $\hat{u}$ - векторное поле, $\hat{u}=\sum_{p=1}^{N} u_{k} \partial_{k}$.

\section{3. ИНТЕРПОЛИРУЮЩИЕ ДИФФЕРЕНЦИАЛЬНЫЕ РЕДУКЦИИ}

Из уравнений Лакса-Сато иерархии (6)

$$
\partial_{n}^{k} \boldsymbol{\Psi}=\widehat{V}_{n}^{k} \boldsymbol{\Psi}, \quad \widehat{V}_{n}^{k}=\sum_{i=0}^{N} V_{n i}^{k} \partial_{i}, \quad V_{n i}^{k}=\left(\left(J_{0}^{-1}\right)_{k i}\left(\Psi^{0}\right)^{n}\right)_{+},
$$

следуют линейные уравнения для якобиана (5):

$$
\partial_{n}^{k} J_{0}=\sum_{i=0}^{N} \partial_{i}\left(V_{n i}^{k} J_{0}\right)
$$

Эти уравнения можно рассматривать как формально сопряженные к линейным уравнениям (9), где сопряженный оператор для $u_{i} \partial_{i}$ определяется как $-\partial_{i} u_{i}$. Интересно заметить, что сопряженные уравнения совпадают с уравнениями (9) только для бездивергентных векторных полей.

Уравнения (10) могут быть записаны также в виде неоднородных линейных уравнений относительно логарифма якобиана:

$$
\partial_{n}^{k} \ln J_{0}=\widehat{V}_{n}^{k} \ln J_{0}+\operatorname{div} \widehat{V}_{n}^{k}, \quad \operatorname{div} \widehat{V}_{n}^{k}=\sum_{i=0}^{N} \partial_{i} V_{n i}^{k} .
$$

Коэффициенты этих уравнений являются полиномами по $\lambda$, функция $\ln J_{0}-\alpha\left(\Psi^{0}\right)^{k}$ есть решение уравнений, следовательно, условие

$$
\left(\ln J_{0}-\alpha\left(\Psi^{0}\right)^{k}\right)_{-}=0
$$

определяет редукцию иерархии (это условие сохраняется в динамике). Подобно случаю иерархии Манакова-Сантини данное условие характеризуется существованием полиномиального решения уравнений (11).

ПРЕДЛОЖЕНИЕ 1. Условие существования полиномиального решения вида

$$
f=-\alpha \lambda^{k}+\sum_{0}^{i=k-2} f_{i}(\mathbf{t}) \lambda^{i}
$$

(где коэффициенты $f_{i}$ не содержат констант) для уравнений (11),

$$
\partial_{n}^{k} f=\widehat{V}_{n}^{k} f+\operatorname{div} \widehat{V}_{n}^{k}
$$

равносильно условию редукиии (12). 
ДокАЗАТЕЛЬство совершенно аналогично доказательству подобного утверждения для иерархии Манакова-Сантини, приведенному в работе [1]. Во-первых, из условия редукции (12) непосредственно следует, что $f=\ln J_{0}-\alpha\left(\Psi^{0}\right)^{k}$ является полиномиальным решением уравнений (11) требуемого вида, таким образом, существование полиномиального решения необходимо для условия редукции.

Для доказательства достаточности заметим, что $F=\ln J_{0}-f$ является решением однородных уравнений (11) (уравнения (9)). Разложим $\lambda$ по степеням $\Psi^{0}$, обращая ряд (3), и представим $F$ в виде

$$
F=\alpha\left(\Psi^{0}\right)^{k}+\sum_{-\infty}^{i=k-2} F_{i}(\mathbf{t})\left(\Psi^{0}\right)^{i}
$$

Нетрудно проверить, что $F$ является решением однородных уравнений (11) тогда и только тогда, когда все коэффициенты $F_{i}(\mathbf{t})$ постоянны. Предполагая, что коэффициенты $f_{i}$ полинома $f$ не содержат констант, мы приходим к заключению, что $\ln J_{0}-\alpha\left(\Psi^{0}\right)^{k}=f$, таким образом, $\left(\ln J_{0}-\alpha\left(\Psi^{0}\right)^{k}\right)_{-}=0$. Предложение доказано.

Еще одна эквивалентная формулировка редукции может быть дана в терминах производящего соотношения (2). Из условия (12) следует, что $J_{0}=e^{\alpha\left(\Psi^{0}\right)^{k}}$, и нетрудно доказать следующее утверждение.

ПреДЛожениЕ 2. Редуцированная иерархия, определяемая производящим соотношением (2) совместно с условием редукции (12), эквивалентна производящему соотношению

$$
\left(e^{-\alpha\left(\Psi^{0}\right)^{k}} d \Psi^{0} \wedge d \Psi^{1} \wedge \cdots \wedge d \Psi^{N}\right)_{-}=0 .
$$

Для вычисления редукций в терминах уравнений (8) удобно начать с неоднородных линейных уравнений (11), соответствующих первым потокам иерархии (7), которые имеют вид

$$
\partial_{1}^{n} f=\left(\lambda \partial_{n}-\sum_{p=1}^{N}\left(\partial_{n} u_{p}\right) \partial_{p}-\left(\partial_{n} u_{0}\right) \partial_{\lambda}\right) f-\partial_{n} \sum_{p=1}^{N} \partial_{p} u_{p}, \quad 1 \leqslant n \leqslant N .
$$

Подставляя $f$ вида (13) в эти уравнения, мы получаем $k$ уравнений на $k-1$ коэффициент $f_{i}$, которые выражают $f_{i}$ через $u_{0}, u_{n}$ и определяют дифференциальную редукцию.

3.1. Случай $k=0$. Бездивергентные векторные поля. При $k=0$ из условия редукции $(12)$, с учетом того, что разложение $J_{0}$ имеет вид $J_{0}=1+J_{0}^{1} \lambda^{-1}+\cdots$ $\left(\right.$ так что $\left.\left(\ln J_{0}\right)_{+}=0\right)$, следует, что $J_{0}=1$. Таким образом, неоднородные линейные уравнения имеют нулевое решение; подставляя его в уравнения, получаем $\operatorname{div} \widehat{V}_{n}^{k}=0$, таким образом, векторные поля в уравнениях Лакса-Сато (9) являются бездивергентными, и потоки иерархии сохраняют объем. Производящее уравнение (2) для редуцированной иерархии имеет вид

$$
\left(d \Psi^{0} \wedge d \Psi^{1} \wedge \cdots \wedge d \Psi^{N}\right)_{-}=0 .
$$

С другой стороны, сохраняющая объем редукция может быть получена из редукции (12) с произвольным $k$ в пределе $\alpha \rightarrow 0$. Таким образом, редукция (12) с произвольным $k$ является интерполирующей между иерархией с сохранением объема и 
иерархией, характеризуемой существованием полиномиального решения уравнений Лакса-Сато (6) (редукция Гельфанда-Дикого).

Для вычисления редукции в терминах уравнений (8) подставляем нулевое решение в уравнения (15) и получаем условие

$$
\operatorname{div} \hat{u}:=\sum_{p=1}^{N} \partial_{p} u_{p}=0 .
$$

Таким образом, уравнения (8) редуцируются к бездивергентным векторным полям $\hat{u}$.

3.2. Случай $k=1$. При $k=1$ условие редукции (12) записывается как $\left(\ln J_{0}-\right.$ $\left.\alpha \Psi^{0}\right)_{-}=0$, таким образом, $\ln J_{0}-\alpha \Psi^{0}=-\left(\alpha \Psi^{0}\right)_{+}$, и для якобиана получаем $J_{0}=$ $e^{\alpha\left(\Psi^{0}-\lambda\right)}$. Производящее соотношение для редуцированной иерархии имеет вид

$$
\left(e^{-\alpha \Psi^{0}} d \Psi^{0} \wedge d \Psi^{1} \wedge \cdots \wedge d \Psi^{N}\right)_{-}=0 .
$$

Редукция подразумевает существование решения $f=-\alpha \lambda$ неоднородных линейных уравнений (11) и справедливость соотношения $\operatorname{div} \widehat{V}_{n}^{k}=\alpha V_{n 0}^{k}$. Используя это равенство для первых потоков (15), вычисляем условие редукции для уравнений (8):

$$
\operatorname{div} \hat{u}:=\sum_{p=1}^{N} \partial_{p} u_{p}=\alpha u_{0} .
$$

С помощью этого условия можно исключить функцию $u_{0}$ из системы $(8)$ и получить редуцированную систему в явном виде:

$$
\partial_{1}^{k} \partial_{q} \hat{u}-\partial_{1}^{q} \partial_{k} \hat{u}+\left[\partial_{k} \hat{u}, \partial_{q} \hat{u}\right]=\alpha^{-1}\left(\left(\partial_{k} \operatorname{div} \hat{u}\right) \partial_{q}-\left(\partial_{q} \operatorname{div} \hat{u}\right) \partial_{k}\right) .
$$

Предел $\alpha \rightarrow 0$ редуцированной иерархии соответствует случаю сохранения объема $(k=0)$, описанному выше, в то время как предел $\alpha \rightarrow \infty$ соответствует иерархии, характеризуемой соотношением $\Psi^{0}=\lambda[10],[18]$. Для этой иерархии векторные поля в уравнениях Лакса-Сато (6) не содержат производной по спектральной переменной, и $u_{0}$ в уравнениях (8) равно нулю,

$$
\partial_{1}^{k} \partial_{q} \hat{u}-\partial_{1}^{q} \partial_{k} \hat{u}+\left[\partial_{k} \hat{u}, \partial_{q} \hat{u}\right]=0
$$

Эта иерархия является "предшественником" гиперкэлеровых иерархий [19], [20], которые соответствуют гамильтоновым векторным полям как в уравнениях Лакса-Сато (6), так и в уравнениях (8). Таким образом, редукция (12) при $k=1$ является интерполирующей между сохраняющей объем иерархией, связанной с системой (8) для бездивергентных векторных полей $\hat{u}$, и предгиперкэлеровой иерархией, связанной с системой (17).

3.3. Случай $k=2$. При $k=2$ условие (12) имеет вид $\left(\ln J_{0}-\alpha\left(\Psi^{0}\right)^{2}\right)_{-}=0$, таким образом, $\ln J_{0}-\alpha\left(\Psi^{0}\right)^{2}=-\alpha\left(\Psi^{0}\right)_{+}^{2}$, и для якобиана получаем $J_{0}=e^{\alpha\left(\Psi^{0}\right)_{-}^{2}}$. Производящее уравнение для редуцированной иерархии записывается как

$$
\left(e^{-\alpha\left(\Psi^{0}\right)^{2}} d \Psi^{0} \wedge d \Psi^{1} \wedge \cdots \wedge d \Psi^{N}\right)_{-}=0 .
$$


Редукция подразумевает существование полиномиального решения $f=-\alpha \lambda^{2}+f_{1}$ неоднородных линейных уравнений (11). Подставляя это решение в уравнения (15), получаем

$$
\partial_{1}^{n} f_{1}=-\left(\partial_{n} \hat{u}\right) f_{1}-\partial_{n} \operatorname{div} \hat{u}, \quad \partial_{n} f_{1}=-2 \alpha\left(\partial_{n} u_{0}\right)
$$

или, исключая $f_{1}$,

$$
\partial_{1}^{n} u_{0}+\left(\partial_{n} \hat{u}\right) u_{0}+\frac{1}{2 \alpha} \partial_{n} \operatorname{div} \hat{u}=0
$$

Эти соотношения представляют собой дифференциальную редукцию для системы (8). Нетрудно проверить, что при подстановке этих соотношений во второе уравнение системы (8) оно удовлетворяется тождественно.

Предел $\alpha \rightarrow 0$ редуцированной иерархии соответствует случаю сохранения объема $(k=0)$, в то время как предел $\alpha \rightarrow \infty$ соответствует иерархии, характеризуемой соотношением $\left(\Psi^{0}\right)_{-}^{2}=0$ или, эквивалентно, $\left(\Psi^{0}\right)^{2}=\lambda^{2}+2 u_{0}$ (редукция Гельфанда-Дикого).

3.4. Случай $k=3$. При $k=3$ условие $(12)$ таково: $\left(\ln J_{0}-\alpha\left(\Psi^{0}\right)^{3}\right)_{-}=0$, и для якобиана получаем $J_{0}=e^{\alpha\left(\Psi^{0}\right)^{3}}$. Производящее уравнение для редуцированной иерархии имеет вид

$$
\left(e^{-\alpha\left(\Psi^{0}\right)^{3}} d \Psi^{0} \wedge d \Psi^{1} \wedge \cdots \wedge d \Psi^{N}\right)_{-}=0
$$

Редукция подразумевает существование полиномиального решения $f=-\alpha \lambda^{3}+$ $f_{1} \lambda+f_{2}$ неоднородных линейных уравнений (11). Подставляя это решение в уравнения (15), получаем

$$
\partial_{1}^{n} f_{2}=-\left(\partial_{n} \hat{u}\right) f_{2}+3 \alpha u_{0}\left(\partial_{n} u_{0}\right)-\partial_{n} \operatorname{div} \hat{u}, \quad \partial_{n} f_{2}=-3 \alpha\left(\partial_{n} \hat{u}\right) u_{0}
$$

или, исключая $f_{2}$,

$$
\partial_{1}^{n}\left(\left(\partial_{n} \hat{u}\right) u_{0}\right)=-\partial_{n}\left(\left(\partial_{n} \hat{u}\right)\left(\partial_{n} \hat{u}\right) u_{0}+u_{0}\left(\partial_{n} u_{0}\right)-\frac{1}{3 \alpha} \partial_{n} \operatorname{div} \hat{u}\right) .
$$

Это соотношение представляет собой дифференциальную редукцию для системы (8).

Предел $\alpha \rightarrow 0$ редуцированной иерархии соответствует случаю сохранения объема $(k=0)$, в то время как предел $\alpha \rightarrow \infty$ соответствует иерархии, характеризуемой соотношением $\left(\Psi^{0}\right)_{-}^{3}=0$ (редукция Гельфанда-Дикого).

\section{4. СЛУЧАЙ $N=2$. СИСТЕМЫ, СВЯЗАННЫЕ СО ВТОРЫМ "НЕБЕСНЫМ" УРАВНЕНИЕМ ПЛЕБАНЬСКОГО}

Мы рассмотрим более подробно конструкцию редукций в случае $N=2$, который содержит второе "небесное” уравнение Плебаньского и его обобщение, предложенное Дунайским [7].

4.1. Случай $k=0$. Редукция сохранения объема отвечает обобщению [10] второго "небесного" уравнения, предложенному Дунайским. Векторные поля в уравнениях (8) суть гамильтоновы (двумерные бездивергентные) поля, и можно записать редуцированную систему (8) в терминах потенциала $\Theta$ так, что $u_{1}=\Theta_{y}, u_{2}=-\Theta_{x}$, 
$x=x^{1}, y=x^{2}$. После введения обозначений $z=-t_{1}^{1}, w=t_{1}^{2}, \phi=u_{0}$ получаем систему Дунайского [7]

$$
\begin{gathered}
\Theta_{w x}+\Theta_{z y}+\Theta_{x x} \Theta_{y y}-\Theta_{x y}^{2}=\phi \\
\phi_{x w}+\phi_{y z}+\Theta_{y y} \phi_{x x}+\Theta_{x x} \phi_{y y}-2 \Theta_{x y} \phi_{x y}=0 .
\end{gathered}
$$

Иерархия, связанная с этой системой, подробно изучалась в работе [10]. Общая система (8) в этих обозначениях имеет вид

$$
\begin{gathered}
\left(\partial_{z y}+\partial_{w x}\right) \hat{u}+\left[\partial_{y} \hat{u}, \partial_{x} \hat{u}\right]=\left(\partial_{y} \phi\right) \partial_{x}-\left(\partial_{x} \phi\right) \partial_{y} \\
\left(\partial_{z y}+\partial_{w x}+\left(\partial_{y} \hat{u}\right) \partial_{x}-\left(\partial_{x} \hat{u}\right) \partial_{y}\right) \phi=0
\end{gathered}
$$

где $\hat{u}=u_{1} \partial_{x}+u_{2} \partial_{y}$.

4.2. Случай $k=1$. Редукция с $k=1$ характеризуется соотношением $J_{0}=$ $e^{\alpha\left(\Psi^{0}-\lambda\right)}$. Производящее уравнение редуцированной иерархии имеет вид

$$
\left(e^{-\alpha \Psi^{0}} d \Psi^{0} \wedge d \Psi^{1} \wedge d \Psi^{2}\right)_{-}=0
$$

Редукция подразумевает существование у неоднородных линейных уравнений (11) решения $f=-\alpha \lambda$. Условие редукции для уравнений (19) имеет вид

$$
\operatorname{div} \hat{u}:=\partial_{x} u_{1}+\partial_{y} u_{2}=\alpha \phi \text {. }
$$

Используя это соотношение, можно исключить функцию $u_{0}$ из системы (19) и получить редуцированную систему в явной форме:

$$
\left(\partial_{z y}+\partial_{w x}\right) \hat{u}+\left[\partial_{y} \hat{u}, \partial_{x} \hat{u}\right]=\alpha^{-1}\left(\left(\partial_{y} \operatorname{div} \hat{u}\right) \partial_{x}-\left(\partial_{x} \operatorname{div} \hat{u}\right) \partial_{y}\right) .
$$

Предел редуцированной иерархии $\alpha \rightarrow 0$ соответствует иерархии системы Дунайского, в то время как предел $\alpha \rightarrow \infty$ соответствует иерархии, характеризуемой соотношением $\Psi^{0}=\lambda[10],[18]$. Для этой иерархии векторные поля в уравнениях Лакса-Сато (6) не содержат производной по спектральной переменной, и $\phi$ в уравнениях (19) равно нулю,

$$
\left(\partial_{z y}+\partial_{w x}\right) \hat{u}+\left[\partial_{y} \hat{u}, \partial_{x} \hat{u}\right]=0 .
$$

Эта иерархия является "предшественником" иерархии второго "небесного" уравнения Плебаньского [18], [19], которое соответствует гамильтоновым векторным полям как в уравнениях Лакса-Сато (6), так и в уравнении (20), сводящемся ко второму "небесному" уравнению Плебаньского

$$
\Theta_{w x}+\Theta_{z y}+\Theta_{x x} \Theta_{y y}-\Theta_{x y}^{2}=0
$$

Таким образом, редукция (12) при $k=1$ является интерполирующей между иерархией, связанной с системой Дунайского (18), и иерархией, связанной с системой (20), которая для гамильтоновых векторных полей сводится ко второму "небесному" уравнению Плебаньского. 
4.3. Случай $k=2$. Редукция при $k=2$ характеризуется соотношением $J_{0}=$ $e^{\alpha\left(\Psi^{0}\right)_{-}^{2}}$. Производящее уравнение редуцированной иерархии имеет вид

$$
\left(e^{-\alpha\left(\Psi^{0}\right)^{2}} d \Psi^{0} \wedge d \Psi^{1} \wedge d \Psi^{2}\right)_{-}=0
$$

Условия редукции в терминах системы (19) записываются как

$$
\begin{gathered}
\partial_{z} \phi-\left(\partial_{x} \hat{u}\right) \phi-\frac{1}{2 \alpha} \partial_{x} \operatorname{div} \hat{u}=0, \\
\partial_{w} \phi+\left(\partial_{y} \hat{u}\right) \phi+\frac{1}{2 \alpha} \partial_{y} \operatorname{div} \hat{u}=0 .
\end{gathered}
$$

Предел $\alpha \rightarrow 0$ соответствует системе Дунайского (18), а предел $\alpha \rightarrow \infty-$ второй редукции Гельфанда-Дикого $\left(\Psi^{0}\right)_{-}^{2}=0$ для системы $(20)$.

4.4. Случай $k=3$. Редукция при $k=3$ характеризуется соотношением $J_{0}=$ $e^{\alpha\left(\Psi^{0}\right)^{3}}$. Производящее уравнение редуцированной иерархии имеет вид

$$
\left(e^{-\alpha\left(\Psi^{0}\right)^{3}} d \Psi^{0} \wedge d \Psi^{1} \wedge d \Psi^{2}\right)_{-}=0
$$

Условия редукции в терминах системы (19) записываются как

$$
\begin{aligned}
\partial_{z}\left(\left(\partial_{x} \hat{u}\right) \phi\right) & =\partial_{x}\left(\left(\partial_{x} \hat{u}\right)\left(\partial_{x} \hat{u}\right) \phi+\phi\left(\partial_{x} \phi\right)-\frac{1}{3 \alpha} \partial_{x} \operatorname{div} \hat{u}\right) \\
\partial_{w}\left(\left(\partial_{y} \hat{u}\right) \phi\right) & =-\partial_{y}\left(\left(\partial_{y} \hat{u}\right)\left(\partial_{y} \hat{u}\right) \phi+\phi\left(\partial_{y} \phi\right)-\frac{1}{3 \alpha} \partial_{y} \operatorname{div} \hat{u}\right) .
\end{aligned}
$$

Предел $\alpha \rightarrow 0$ соответствует системе Дунайского (18), а предел $\alpha \rightarrow \infty$ - третьей редукции Гельфанда-Дикого для системы (20).

\section{5. ХАРАКТЕРИЗАЦИЯ РЕДУКЦИЙ В ТЕРМИНАХ ДАННЫХ ОДЕВАНИЯ}

Схема одевания иерархии (2), (6) может быть сформулирована в терминах $(N+1)$ компонентной нелинейной задачи Римана-Гильберта на единичной окружности $S$ в плоскости комплексной переменной $\lambda$ :

$$
\begin{aligned}
& \Psi_{\text {in }}^{0}=F_{0}\left(\Psi_{\text {out }}^{0}, \Psi_{\text {out }}^{1}, \ldots, \Psi_{\text {out }}^{N}\right), \\
& \Psi_{\text {in }}^{k}=F_{k}\left(\Psi_{\text {out }}^{0}, \Psi_{\text {out }}^{1}, \ldots, \Psi_{\text {out }}^{N}\right), \quad 1 \leqslant k \leqslant N,
\end{aligned}
$$

где функции $\Psi_{\text {in }}^{0}(\lambda, \mathbf{t}), \Psi_{\text {in }}^{k}(\lambda, \mathbf{t})$ аналитичны внутри единичной окружности, функции $\Psi_{\text {out }}^{0}(\lambda, \mathbf{t}), \Psi_{\text {out }}^{k}(p, \mathbf{t})$ аналитичны вне единичной окружности и имеют разложение вида $(3),(4)$. Предполагается, что функции $F_{0}, F_{k}$ определяют (по крайней мере локально) диффеоморфизм пространства $\mathbb{C}^{N+1}, \mathbf{F} \in \operatorname{Diff}(N+1)$, и мы называем их данными одевания. В компактной форме задачу (21) можно записать как

$$
\boldsymbol{\Psi}_{\text {in }}=\mathbf{F}\left(\boldsymbol{\Psi}_{\text {out }}\right) \text {. }
$$

Можно непосредственно показать, что задача (21) влечет аналитичность дифференциальной формы

$$
\Omega_{0}=J_{0}^{-1} d \Psi^{0} \wedge d \Psi^{1} \wedge \cdots \wedge d \Psi^{N}
$$


(где независимые переменные дифференциала включают все времена $\mathbf{t}$ и $\lambda$ ) в комплексной плоскости и производящее соотношение (2), определяя таким образом решение иерархии. Рассматривая редукцию к группе сохраняющих объем диффеоморфизмов $\operatorname{SDiff}(N+1)$, мы получаем редукцию общей иерархии $(2)$ к случаю $J_{0}=1$ (бездивергентные векторные поля),

$$
\left(d \Psi^{0} \wedge d \Psi^{1} \wedge \cdots \wedge d \Psi^{N}\right)_{-}=0 .
$$

При $N=2$ мы имеем иерархию системы Дунайского.

Для построения класса редукций (12) необходимо рассмотреть нечто типа "подкрученного" условия сохранения объема. Пусть функции $G_{q}\left(y_{0}, \ldots, y_{N}\right), 0 \leqslant q \leqslant N$, определяют сохраняющий объем диффеоморфизм $\mathbf{G} \in \operatorname{SDiff}(N+1)$,

$$
\left|\frac{D\left(G_{0}, \ldots, G_{N}\right)}{D\left(y_{0}, \ldots, y_{N}\right)}\right|=1
$$

где для якобиана мы используем обозначение

$$
\left|\frac{D\left(y_{1}, \ldots, y_{N}\right)}{D\left(x_{1}, \ldots, x_{N}\right)}\right|=\operatorname{det} \frac{D\left(y_{1}, \ldots, y_{N}\right)}{D\left(x_{1}, \ldots, x_{N}\right)}=\operatorname{det}\left(\frac{\partial y_{i}}{\partial x_{j}}\right) \text {. }
$$

Зафиксируем набор аналитических функций $f_{q}\left(y_{0}, \ldots, y_{n}\right)$ (данные редукции), определяющих диффеоморфизм, и рассмотрим задачу

$$
\mathbf{f}\left(\boldsymbol{\Psi}_{\text {in }}\right)=\mathbf{G}\left(\mathbf{f}\left(\boldsymbol{\Psi}_{\text {out }}\right)\right),
$$

которая соответствует редукции иерархии. Условие редукции в терминах данных одевания для задачи (22) имеет вид

$$
\mathbf{f} \circ \mathbf{F} \circ \mathbf{f}^{-1} \in \operatorname{SDiff}(N+1) .
$$

Это условие мы и называем "подкрученным" условием сохранения объема. В терминах уравнений иерархии редукция характеризуется соотношением

$$
\left(d f_{0}(\boldsymbol{\Psi}) \wedge \cdots \wedge d f_{N}(\boldsymbol{\Psi})\right)_{\text {in }}=\left(d f_{0}(\boldsymbol{\Psi}) \wedge \cdots \wedge d f_{N}(\boldsymbol{\Psi})\right)_{\mathrm{out}},
$$

таким образом, функция

$$
\Omega_{\text {red }}=d f_{0}(\boldsymbol{\Psi}) \wedge \cdots \wedge d f_{N}(\boldsymbol{\Psi})
$$

аналитична в комплексной плоскости, и редуцированная иерархия определяется производящим соотношением $\left(d f_{0}(\boldsymbol{\Psi}) \wedge \cdots \wedge d f_{N}(\boldsymbol{\Psi})\right)_{-}=0$ или, эквивалентно,

$$
\left(\left|\frac{D\left(f_{0}, \ldots, f_{N}\right)}{D\left(\Psi^{0}, \ldots, \Psi^{N}\right)}\right| d \Psi^{0} \wedge d \Psi^{1} \wedge \cdots \wedge d \Psi^{N}\right)_{-}=0 .
$$

Диффеоморфизм f для редуцированной иерархии определен по модулю сохраняющего объем диффеоморфизма. Чтобы получить редуцированную иерархию (14), можно использовать симметричный выбор

$$
f_{0}(\boldsymbol{\Psi})=\Psi^{0}, \quad f_{n}(\boldsymbol{\Psi})=e^{-\alpha N^{-1}\left(\Psi^{0}\right)^{k}} \Psi^{n}, \quad 1 \leqslant n \leqslant N .
$$

Таким образом, мы приходим к следующему заключению.

ПреДЛОЖЕНИЕ 3. В терминах даннъх одевания для задачи (22) класс редукиий (12) характеризуется условием (24), где $\mathbf{f}$ определяется формулой (25). 
Благодарности. Автор благодарен С.В. Манакову за полезные обсуждения. Исследование было частично поддержано РФФИ (гранты № 09-01-92439-КЭ_а, 10-01-00787_а) и Программой поддержки ведущих научных школ (грант НШ-4887. 2008.2).Участие автора в конференции "Нелинейная физика: теория и эксперимент. VI", где был представлен доклад, из которого возникла эта статья, стало возможным благодаря РФФИ (грант № 10-01-08122-з) и гостеприимству организаторов.

\section{Список литературы}

[1] L. V. Bogdanov, J. Phys. A, 43:11 (2010), 115206, 11 pp., arXiv: 0910.4004.

[2] S. V. Manakov, P. M. Santini, Писъма в ЖЭТФ, 83:10 (2006), 534-538, arXiv: nlin/0604023.

[3] С. В. Манаков, П. М. Сантини, ТМФ, 152:1 (2007), 147-156.

[4] S. V. Manakov, P. M. Santini, J. Phys. A, 41:5 (2008), 055204, 23 pp., arXiv: 0707.1802.

[5] M. Dunajski, J. Phys. A, 41:31 (2008), 315202, 9 pp., arXiv: 0804.1234.

[6] M. V. Pavlov, J. Math. Phys., 44:9 (2003), 4134-4156, arXiv: nlin/0301010.

[7] M. Dunajski, J. Geom. Phys., 51:1 (2004), 126-137, arXiv: nlin/0311024.

[8] L. Martinez Alonso, A. B. Shabat, Phys. Lett. A, 300:1 (2002), 58-64.

[9] Л. Мартинес Алонсо, А. Б. Шабат, ТМФ, 140:2 (2004), 216-229, arXiv: nlin/0312043.

[10] L. V. Bogdanov, V.S. Dryuma, S. V. Manakov, J. Phys. A, 40:48 (2007), 14383-14393, arXiv: 0707.1675.

[11] Л. В. Богданов, ТMФ, 160:1 (2009), 15-22, arXiv: 0810.2397.

[12] J.F. Plebański, J. Math. Phys., 16:12 (1975), 2395-2402.

[13] M. Dunajski, Proc. R. Soc. Lond. Ser. A, 458:2021 (2002), 1205-1222, arXiv: math/0102225.

[14] M. Jimbo, T. Miwa, Publ. Res. Inst. Math. Sci., 19:3 (1983), 943-1001.

[15] V.E. Zakharov, Duke Math. J., 94:1 (1998), 103-139.

[16] В. Е. Захаров, С. В. Манаков, Докл. РАН, 360:3 (1998), 324-327.

[17] L. V. Bogdanov, E. V. Ferapontov, J. Geom. Phys., 52:3 (2004), 328-352.

[18] L. V. Bogdanov, B. G. Konopelchenko, Phys. Lett. A, 345:1-3 (2005), 137-143, arXiv: nlin/0504062.

[19] K. Takasaki, J. Math. Phys., 30:7 (1989), 1515-1521.

[20] K. Takasaki, J. Math. Phys., 31:8 (1990), 1877-1888. 